\title{
Serum proton NMR metabolomics analysis of human lung cancer following microwave ablation
}

\author{
Jian-Ming $\mathrm{Hu}^{*}$ and Huang-Tao Sun
}

\begin{abstract}
Background: To find potential serum biomarkers of microwave ablation (MWA) for treatment of human lung cancer by ${ }^{1} \mathrm{H}$ nuclear magnetic resonance (NMR)-based metabolomics analysis.

Methods: Serum specimens collected from 43 healthy individuals, 39 patients with advanced non-small cell lung cancer (NSCLC) and 38 NSCLC patients treated with MWA, were subjected to ${ }^{1} \mathrm{H}$ NMR-based metabolomics analysis. Partial least squares discriminant analysis was used to analyze the data.

Results: Compared with healthy controls, NSCLC patients showed significantly elevated serum levels of lactate, alanine, glutamate, proline, glycoprotein, phenylalanine, tyrosine and tryptophan, and markedly decreased serum levels of glucose, taurine, glutamine, glycine, phosphocreatine and threonine $(p<0.05)$. MWA treatment reversed the metabolic profiles of NSCLC patients towards the control group.

Conclusions: ${ }^{1} \mathrm{H}$ NMR-based metabolomics analysis enhanced the current understanding of the mechanisms involved in NSCLC, and uncovered the therapeutic potential of MWA for treatment of NSCLC. The above disturbed serum metabolites were proposed to be the potential biomarkers that may help to predict NSCLC and to evaluate the efficacy of MWA in the treatment of NSCLC.
\end{abstract}

Keywords: Non-small cell lung cancer, Microwave ablation, Nuclear magnetic resonance, Metabolomics, PLS-DA

\section{Background}

In China, lung cancer is the most common incident cancer and the leading cause of cancer death for both men and women in 2015 [1]. It was reported that 486,555 patients died from lung cancer and the 5 year survival rate for patients was less than $20 \%$ in 2010 [2]. Surgery is the most preferred and effective method for lung cancer patients. However, many patients are inoperable such as advanced non-small cell lung cancer patients and lung metastases patients. At present, minimally invasive thermal therapy including radiofrequency ablation (RFA) and microwave ablation (MWA) are widely used in lung cancer patients in clinic [3], which use heat generated by the biological effects of tumor cells directly to cause irreversible damage or coagulation necrosis in tumor tissues [4].

\footnotetext{
* Correspondence: hu.jianming@aliyun.com

Thoracic surgeons, Jiangxi Provincial People's Hospital, 152 Patriotic Road, Nanchang City 333000, People's Republic of China
}

MWA has several advantages such better heating of larger tumour volumes, which is consider to be more suitable for lung tissues [5]. Therapeutic effect evaluation of MWA is very important for doctors to easily and timely adjust the treatment plan to maximize the efficacy of thermal ablation therapy. Efficacy evaluation of thermal ablation is currently based on the anatomical imaging, morphology or pathology examination. The major disadvantages of these traditional indicators include poor sensitivity and specificity, which cannot effectively estimate the curative effects and predict prognosis of NSCLC patients [6].

A number of NMR-based metabolomics studies have reported biomarkers that effectively discriminate between NSCLC subjects and healthy controls [7-9]. Deja and co-workers proposed that the the following metabolite biomarkers could potentially be useful in distinguishing lung cancer states: isoleucine, acetoacetate, and creatine as well as the two NMR signals of $\mathrm{N}$-acetylated 
glycoproteins and glycerol [10]. Rocha investigated the metabolic profile variations of plasma from lung cancer patients and healthy controls through NMR-based metabolomics. Relatively lower high density lipoprotein (HDL) and higher very low density lipoprotein (VLDL) and low-density lipoprotein (LDL) in the patients' plasma, together with increased lactate and pyruvate and decreased levels of glucose, citrate, formate, acetate, several amino acids and methanol, were detected [11]. In another study of temporal characterization of serum metabolite signatures in lung cancer patients undergoing chemotherapy \pm radiation treatment, the feasibility of metabolites in the plasma of lung cancer patients as temporal biomarkers of clinical outcomes were discussed [12]. However, few studies take advantage of NMR spectroscopy's ability to obtain a metabolic fingerprint of NSCLC patients after MWA treatment.

To further investigate the overall metabolic profiling of NSCLC patients and the efficacy of microwave thermal ablation in the treatment of NSCLC, metabolomics approach was introduced to give a holistic view of endogenous metabolites of the patients, which could deepen our knowledge about non-small cell lung cancer and promote the thermal ablation treatment for lung cancer, and these should be benefit for human health in the future.

\section{Methods}

\section{Ethics statement}

The study was approved by the Institutional Ethics Committee of the Jiangxi Provincial People's Hospital. A written informed consent was obtained from all participants involved in this study.

\section{Patients and samples}

Between 20 February 2014 and 30 May 2016 patients who met the following criteria were retrospectively enrolled in the study: (1) pathologically verified peripheral NSCLC, (2) stage IIIB or IV, (3) chemotherapy-naive (except patients with recurrence treated with adjuvant chemotherapy or adjuvant radiation), (4) an Eastern Cooperation Oncology Group (ECOG) performance status of 0 to 2, and (5) adequate pulmonary, cardiac, hepatic, renal and hematological functions to allow anticancer treatment.

Selected characteristics of the NSCLC cases and controls were summarized in Table 1. Blood samples were collected prior to the morning meal, and serums were obtained by centrifugation of the blood samples at $3500 \mathrm{rpm}$ for $10 \mathrm{~min}$ at $4{ }^{\circ} \mathrm{C}$. Aliquots of the serum samples were stored at $-80^{\circ} \mathrm{C}$ until NMR analysis.

\section{Serum preparation and ${ }^{1} \mathrm{H}$ NMR spectroscopy}

About $1 \mathrm{~mL}$ serum samples were deproteinized by methanol with the ratios of serum: methanol as $1: 2(\mathrm{v} / \mathrm{v})$.
Table 1 Clinicopathological characteristics of the enrolled NSCLC patients, MWA treated patients and healthy controls

\begin{tabular}{|c|c|c|c|}
\hline Characteristic & NSCLC & MWA & Healthy \\
\hline Number & 38 & 39 & 43 \\
\hline \multicolumn{4}{|l|}{ Gender } \\
\hline Male & 19 & 20 & 22 \\
\hline Female & 19 & 19 & 21 \\
\hline \multicolumn{4}{|l|}{ Age (years) } \\
\hline$\geq 60$ & 21 & 20 & 21 \\
\hline$<60$ & 17 & 19 & 22 \\
\hline \multicolumn{4}{|l|}{ Stage } \\
\hline$\| \mathrm{IIB}$ & 10 & 13 & - \\
\hline IV & 28 & 26 & - \\
\hline \multicolumn{4}{|l|}{ Tumour location } \\
\hline Peripheral & 38 & 39 & - \\
\hline Central & 0 & 0 & - \\
\hline \multicolumn{4}{|l|}{ Tumour size $(\mathrm{cm})$} \\
\hline$\geq 3.5$ & 21 & 22 & - \\
\hline$<3.5$ & 17 & 17 & - \\
\hline \multicolumn{4}{|l|}{ ECOG performance status } \\
\hline 0 & 0 & 2 & - \\
\hline 1 & 36 & 35 & - \\
\hline 2 & 2 & 2 & - \\
\hline \multicolumn{4}{|l|}{ Pathology } \\
\hline Adenocarcinoma & 19 & 20 & - \\
\hline Squamous carcinoma & 13 & 10 & - \\
\hline Adenosquamous carcinoma & 6 & 8 & - \\
\hline Large cell carcinoma & 0 & 1 & - \\
\hline MWA time (min), mean (range) & - & $14.0(4.0-42.0)$ & - \\
\hline \multicolumn{4}{|l|}{ MWA power } \\
\hline 70 & - & 33 & - \\
\hline 60 & - & 6 & - \\
\hline \multicolumn{4}{|l|}{ Number of antennas } \\
\hline One & - & 16 & - \\
\hline Two & - & 23 & - \\
\hline
\end{tabular}

The mixtures were vortexed and incubated at $-20{ }^{\circ} \mathrm{C}$ for about $30 \mathrm{~min}$, and then were centrifuged into pellet proteins at $12000 \mathrm{~g}$ for $30 \mathrm{~min}$. The supernatants were transferred into fresh tubes and lyophilized. The dried samples were dissolved in $600 \mu \mathrm{L} 99.8 \% \mathrm{D}_{2} \mathrm{O}$ phosphate buffer $(0.2 \mathrm{M}, \mathrm{pH}$ at 7.0$)$ containing $0.05 \%$ sodium salt of 3-trimethylsilylpropionic acid (TSP, $w / v$ ), vortexed, centrifuged and decanted to $5 \mathrm{~mm}$ NMR tubes.

All ${ }^{1} \mathrm{H}$ NMR spectra were recorded with a Bruker AV $500 \mathrm{MHz}$ spectrometer. A transverse relaxation-edited Carr-Purcell-Meiboom-Gill sequence [recycle delay-90$(\tau-180-\tau) n$-acquisition] with a total spin echo delay (2nt) 
of $40 \mathrm{~ms}$ was used to attenuate broad signals from slowly tumbling molecules such as proteins, whereupon the signals of the micro-molecule metabolites were clearly observed. ${ }^{1} \mathrm{H}$ NMR spectra were measured with 128 scans into $64 \mathrm{~K}$ data points with a recycle delay of $3 \mathrm{~s}$ over a spectral width of $20 \mathrm{ppm}$. The spectra were Fourier-transformed after multiplication by an exponential window function corresponding to a line broadening of $0.5 \mathrm{~Hz}$. Resonances were assigned by querying the database HMDB (http://www.hmdb.ca/), MMCD (http:// mmcd.nmrfam.wisc.edu/), and were aided by the Chenomx NMR suite (version 8.0, Chenomx, Inc.).

\section{Data processing and multivariate analysis}

The ${ }^{1} \mathrm{H}$ NMR spectra were manually phased and baselinecorrected by MestReNova (version 8.0.1,Mestrelab Research $\mathrm{SL})$. Spectra were aligned to the TSP signal at zero ppm. A linear interpolation method was used to align the spectra using MestReNova. Regions containing residual water and methanol signals were removed, and the spectra were binned into integrated segments with equal widths of $0.01 \mathrm{ppm}$ and adjusted by probabilistic quotient normalization and paretoscaling prior to multivariate analysis.

First, an unsupervised principal component analysis (PCA) was used for for the metabolomics data overview and the spotting of outliers, and then for the detection of any grouping. Clustering was failed for the dataset (Additional file 1: Figure S1). Then a supervised partial least squares discriminant analysis (PLS-DA) was used to gain valuable insights on group-predictive spectral features. The metabolic profiles could be visualized as score plot, where each point represents a sample. The corresponding loading plot and S-plot were generated to provide information on the metabolites that influence clustering of the samples. In addition, a correlation circle plot produced by sparse PLS regression was applied to illustrate the relationships between certain integral metabolites and groupings.

To quantitatively assess the performance of the model, a repeated two fold cross-validation and permutation testing was carried out. Permutation distribution of the test statistic was computed based on 10,000 times repeated random permutation of the class labels and the significance value was taken to be the fraction of samples not exceeding the test statistic for the original sample. The overall quality of the model was evaluated by the cumulative $R^{2}$, while the predictive ability was assessed by the cumulative $\mathrm{Q}^{2}$. Integration areas of the detected metabolites with marked differentiating ability were first tested for distribution normality. The student t-test or rank test was then employed to detect differences in metabolite levels between groups and $p<0.05$ was considered to be statistically significant.

\section{Pathway analysis}

Differential metabolites were subjected to pathway analysis by MetaboAnalyst [13], which combines results from powerful pathway enrichment analysis with the pathway topology analysis, to identify the most relevant pathways involved in the NSCLC patients.

In order to excavate anything gene as potential biomarkers, and to integrate gene and metabolomics information to provide a better understanding of the efficacy of MWA in the treatment of NSCLC, the identified metabolites in metabolomics were then mapped to the KEGG pathway for biological interpretation of higherlevel systemic functions. The metabolites and corresponding pathways were visualized using KEGG Mapper tool (http://www.genome.jp/kegg/mapper.html).

\section{Results}

${ }^{1} \mathrm{H}$ NMR spectra

Representative ${ }^{1} \mathrm{H}$ NMR spectra of serum samples were shown in Fig. 1, with metabolites assigned. The variations in serum metabolites between groups were summarized in Table 2. The means and standard deviation values of metabolites for each group were provided in the Additional file 1: Table S1. Compared with the control group, the NSCLC group showed elevated levels of lactate, alanine, glutamate, proline, glycoprotein, phenylalanine, tyrosine and tryptophan, and decreased levels of glucose, taurine, glutamine, glycine, phosphocreatine and threonine, which could be partially or completely reversed by MWA treatment.

\section{Multivariate analysis}

The validity of the PLS-DA model against over-fitting was assessed by the parameters $R^{2}(0.68)$, and the predictive ability was described by $\mathrm{Q}^{2}(0.45)$. Theoretically, the closer the $\mathrm{R}^{2}$ and $\mathrm{Q}^{2}$ value to 1 , the better the PLSDA model is. A permutation test $(n=1000)$ was then performed to assure the predictive capacity of the PLSDA model. The observed statistic $P$ values via permutation testing were 0.036 which was less than 0.05 , thus confirming the validity of the PLS-DA model. The PLSDA score plot (Fig. 2) revealed satisfactory discrimination between the three groups. MWA treatment could reverse the disturbed metabolic profile towards the control group. The corresponding color-coded coefficient loading plot on the first component (Fig. 3) and S-plot (Fig. 4) visualized the contribution of each metabolite to the separation between groups. Significantly altered metabolites between groups were the pseudo peaks in warm color in the loadings plot, and those points in the upper right and lower left quadrants of the S-plot. Peaks in the positive axis and points in the upper right quadrants means the metabolites increased in the NSCLC group. The select metabolites were graphed as scatter 


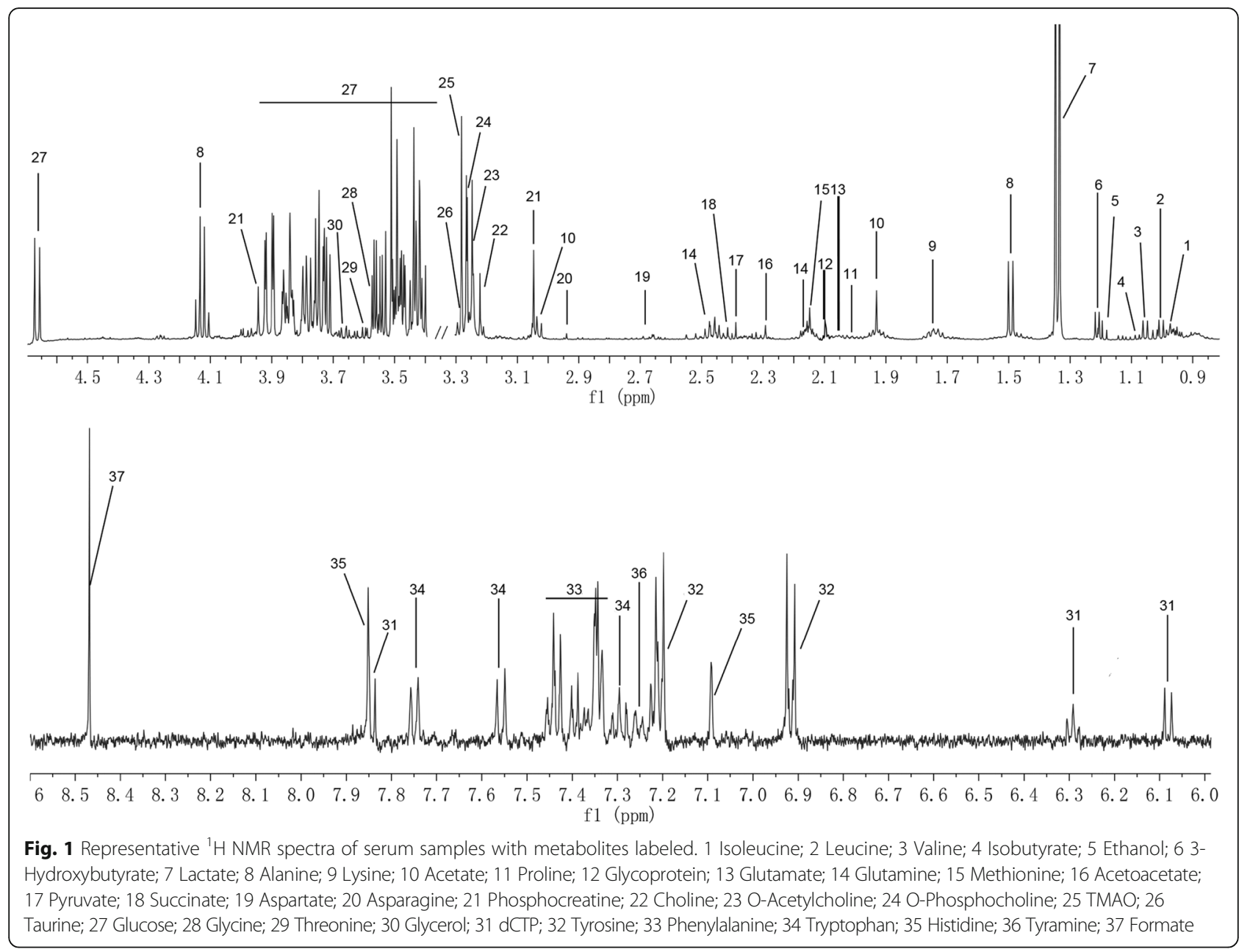

plots in order to show the interindividual variation (Additional file 1: Figure S2). A concentric correlation circle concerning the correlations between the identified $\mathrm{m}$ a status similar to healthy controls etabolites and the three groups were plotted as scatter plot, provided additional information on the endogenous metabolites among groupings (Fig. 5).

\section{Network visualization of metabolites and pathways}

The evidently disturbed metabolites were subjected to pathway analysis using a web-tool MetaboAnalyst [13]. Consequently, four metabolic pathways, including taurine and hypotaurine metabolism; $\mathrm{d}$-glutamine and $\mathrm{d}$ glutamate metabolism; glycine, serine and threonine metabolism; alanine, aspartate and glutamate metabolism, were filtered out as the most important pathways related with the metabolic disturbances in NSCLC patients (Fig. 6). The $P$ values table generated by MetaboAnalyst in association with Fig. 6 was provided in the Additional file 1: Table S2. These metabolic alterations and the associated pathways provided insights into the mechanisms involved in the development and progression of NSCLC.

The identified metabolites were mapped on the KEGG reference pathway diagram concerning central carbon metabolism in cancer (Fig. 7), where red and blue nodes represented increased and decreased metabolites in NSCLC patients, respectively. Some oncogene and tumor suppressor gene were found, such as the tumor suppressor gene of p53 [14] SIRT3 [15] and SIRT6 [16], and oncogene of Ras [17] PI3K [18], AKT [19] and c-Myc [20]. However, these genes were not found via any genetic exploration in this study, but are instead potentially linked to the measured downstream metabolic changes, which might reflect the potential treatment mechanism of MWA on NSCLC.

\section{Discussion}

Lung cancer is a major contributor to cancer-related mortality and burden of disease. MWA can be used to provide potentially curative tumour ablation in patients who are not candidates for surgical resection [21]. The 
Table 2 Potential serum biomarkers identified by ${ }^{1} \mathrm{H}$ NMR and their variations among NSCLC patients, MWA treated patients and healthy controls

\begin{tabular}{|c|c|c|c|c|c|c|c|c|}
\hline \multirow[t]{2}{*}{ No. } & \multirow[t]{2}{*}{ Metabolites } & \multirow[t]{2}{*}{ Chemical shifts (ppm) } & \multicolumn{2}{|c|}{ NSCLC/CTRL } & \multicolumn{2}{|c|}{ MWA/NSCLC } & \multicolumn{2}{|c|}{ MWA/CTRL } \\
\hline & & & fold & p & fold & $p$ & fold & $p$ \\
\hline 1 & Isoleucine & $0.94(t), 1.00(d)$ & 1.09 & & 1.18 & & 1.29 & * \\
\hline 2 & Leucine & $0.96(d), 0.97(d)$ & 1.09 & & 0.93 & & 1.00 & \\
\hline 3 & Valine & $0.99(d), 1.05(d)$ & 1.11 & & 1.17 & & 1.30 & $* *$ \\
\hline 4 & Isobutyrate & 1.07 (d) & 1.06 & & 1.09 & & 1.16 & \\
\hline 5 & Ethanol & $1.19(d), 3.65(q)$ & 1.01 & & 1.11 & & 1.12 & \\
\hline 6 & 3-Hydroxybutyrate & $1.20(\mathrm{~d}), 2.30,2.41(\mathrm{~m})$ & 1.15 & & 1.09 & & 1.25 & $* * *$ \\
\hline 7 & Lactate & $1.33(d), 4.12(q)$ & 2.13 & $* * *$ & 0.90 & * & 1.92 & $* * *$ \\
\hline 8 & Alanine & $1.48(d), 3.78(q)$ & 1.76 & $* * *$ & 0.87 & * & 1.52 & *** \\
\hline 9 & Lysine & $1.73(\mathrm{~m}), 1.91(\mathrm{~m}), 3.30(\mathrm{t})$ & 1.12 & & 1.10 & & 1.24 & $* * *$ \\
\hline 10 & Acetate & $1.92(\mathrm{~s})$ & 1.18 & & 1.08 & & 1.27 & $* *$ \\
\hline 11 & Proline & $2.01(\mathrm{~m})$ & 1.45 & $* *$ & 0.86 & & 1.25 & $* *$ \\
\hline 12 & Glycoprotein & $2.09(\mathrm{~s})$ & 1.18 & * & 0.89 & & 1.05 & \\
\hline 13 & Glutamate & $2.05(\mathrm{~m}), 2.12(\mathrm{~m}), 2.36(\mathrm{~m})$ & 1.14 & $* *$ & 0.85 & $* * *$ & 0.96 & \\
\hline 14 & Glutamine & $2.14(\mathrm{~m}), 2.45(\mathrm{~m})$ & 0.80 & $* * *$ & 1.11 & & 0.89 & * \\
\hline 15 & Methionine & $2.14(\mathrm{~s}), 2.65(\mathrm{t})$ & 1.10 & & 1.11 & & 1.22 & * \\
\hline 16 & Acetoacetate & $2.28(s)$ & 1.15 & & 1.19 & & 1.37 & $* *$ \\
\hline 17 & Pyruvate & $2.38(s)$ & 1.19 & & 0.85 & & 1.01 & \\
\hline 18 & Succinate & $2.41(s)$ & 1.21 & & 0.81 & & 0.98 & \\
\hline 19 & Aspartate & $2.68,2.81(\mathrm{~m})$ & 0.86 & & 1.17 & & 1.01 & \\
\hline 20 & Asparagine & $2.87,2.95(\mathrm{~m})$ & 0.88 & & 1.16 & & 1.02 & \\
\hline 21 & Phosphocreatine & $3.04(\mathrm{~s}), 3.93$ (s) & 0.81 & $* * *$ & 1.13 & & 0.91 & \\
\hline 22 & Choline & $3.20(s)$ & 0.89 & & 1.07 & & 0.95 & \\
\hline 23 & O-Acetylcholine & $3.21(\mathrm{~s})$ & 0.89 & & 0.89 & & 0.79 & ** \\
\hline 24 & O-Phosphocholine & $3.23(\mathrm{~s})$ & 0.89 & & 0.86 & & 0.76 & $* * *$ \\
\hline 25 & TMAO & $3.27(s)$ & 1.07 & & 0.89 & & 0.95 & \\
\hline 26 & Taurine & $3.27(\mathrm{t}), 3.42(\mathrm{t})$ & 0.76 & $* * *$ & 1.38 & $* * *$ & 1.05 & \\
\hline 27 & Glucose & 3.4-3.92 (m) & 0.92 & * & 1.08 & * & 0.99 & \\
\hline 28 & Glycine & $3.56(s)$ & 0.89 & * & 1.12 & * & 1.00 & \\
\hline 29 & Threonine & $3.59(\mathrm{~d}), 4.25(\mathrm{~m})$ & 0.82 & * & 1.20 & $* *$ & 0.98 & \\
\hline 30 & Glycerol & $3.58,3.65(\mathrm{~m})$ & 1.24 & & 0.82 & & 1.01 & \\
\hline 31 & $\mathrm{dCTP}$ & $6.07(d), 6.08(t), 7.83(d)$ & 0.86 & & 0.86 & & 0.74 & $* * *$ \\
\hline 32 & Tyrosine & $6.91(d), 7.20(d)$ & 1.16 & $* *$ & 0.97 & & 1.12 & $* *$ \\
\hline 33 & Phenylalanine & $7.33(\mathrm{~d}), 7.38(\mathrm{t}), 7.43(\mathrm{t})$ & 1.17 & * & 0.83 & & 0.97 & \\
\hline 34 & Tryptophan & $7.55(d), 7.74(d)$ & 1.30 & $* * *$ & 0.89 & & 1.15 & $* *$ \\
\hline 35 & Histidine & 7.08 (s), 7.84 (s) & 0.88 & & 1.14 & & 1.00 & \\
\hline 36 & Tyramine & 7.24 (d) & 0.88 & & 0.96 & & 0.85 & $* *$ \\
\hline 37 & Formate & $8.46(s)$ & 0.87 & & 1.12 & & 0.98 & \\
\hline
\end{tabular}

${ }^{1} \mathrm{H}-\mathrm{NMR} 1 \mathrm{H}$-nuclear magnetic resonance, NSCLC non-small-cell lung cancer, MWA microwave ablation, TMAO trimethylamine $\mathrm{N}$-oxide. Asterisk $\mathrm{P}<0.05$, double asterisk $P<0.01$ and triple asterisk $P<0.001$. Multiplicity: $\mathrm{s}$ singlet, $\mathrm{d}$ doublet, $\mathrm{t}$ triplet, $\mathrm{m}$ multiplets

metabolic signature of serum from MWA treated NSCLC patients and underlying mechanisms has not been evaluated holistically. In this study, metabolic profile analysis was employed to extravagate the underlying mechanisms of MWA as a treatment of NSCLC. In serum of NSCLC patients, levels of lactate, alanine, glutamate, proline, glycoprotein, phenylalanine, tyrosine and tryptophan were increased, while levels of glucose, 

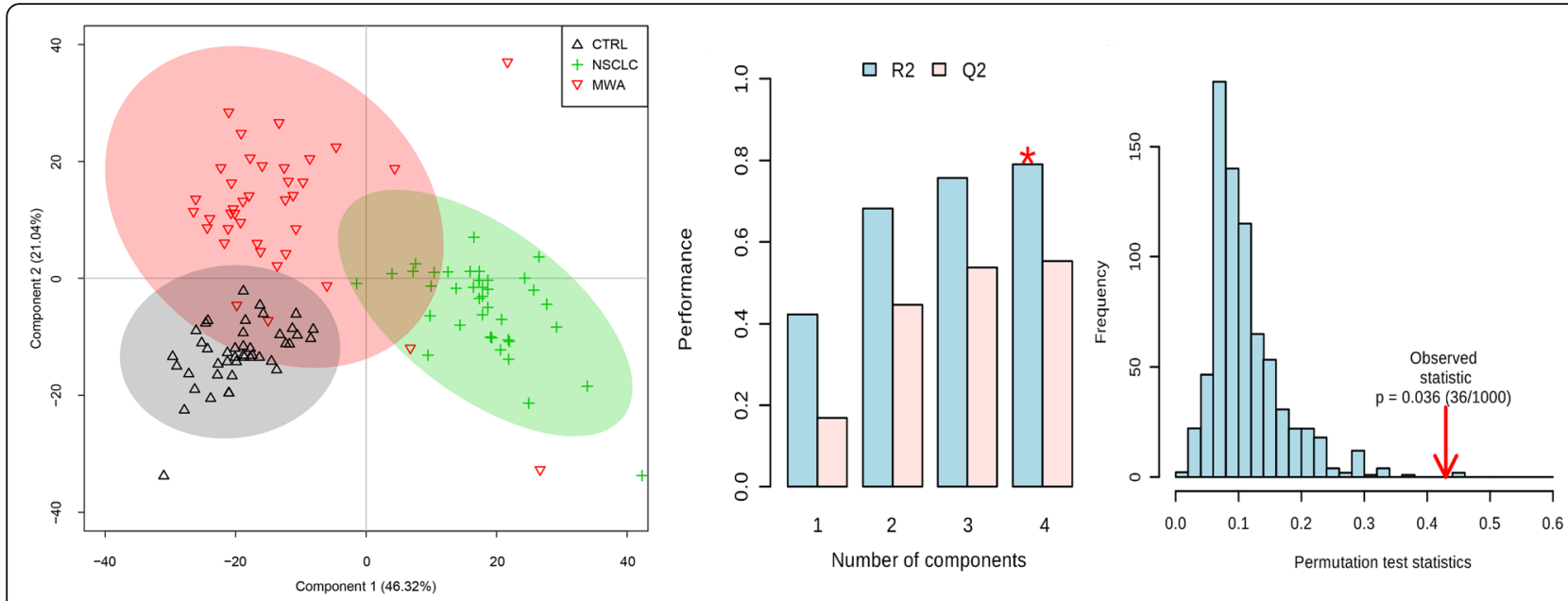

Fig. 2 Scores plot, cross validation and a permutation test of PLS-DA model for CTRL, NSCLC and MWA groups. CTRL, healthy controls ( $n=43$ ). NSCLC, nonsmall-cell lung cancer patients $(n=38)$. MWA, microwave ablation treated patients $(n=38)$. The values of $R^{2}(0.68)$ and $Q^{2}(0.45)$ revealed satisfactory goodness of fit and goodness of prediction, respectively. The nominal $P$ value $(0.036)$ of the permutation test was less than 0.05 , confirming the validity of the PLS-DA model at a $95 \%$ confidence level

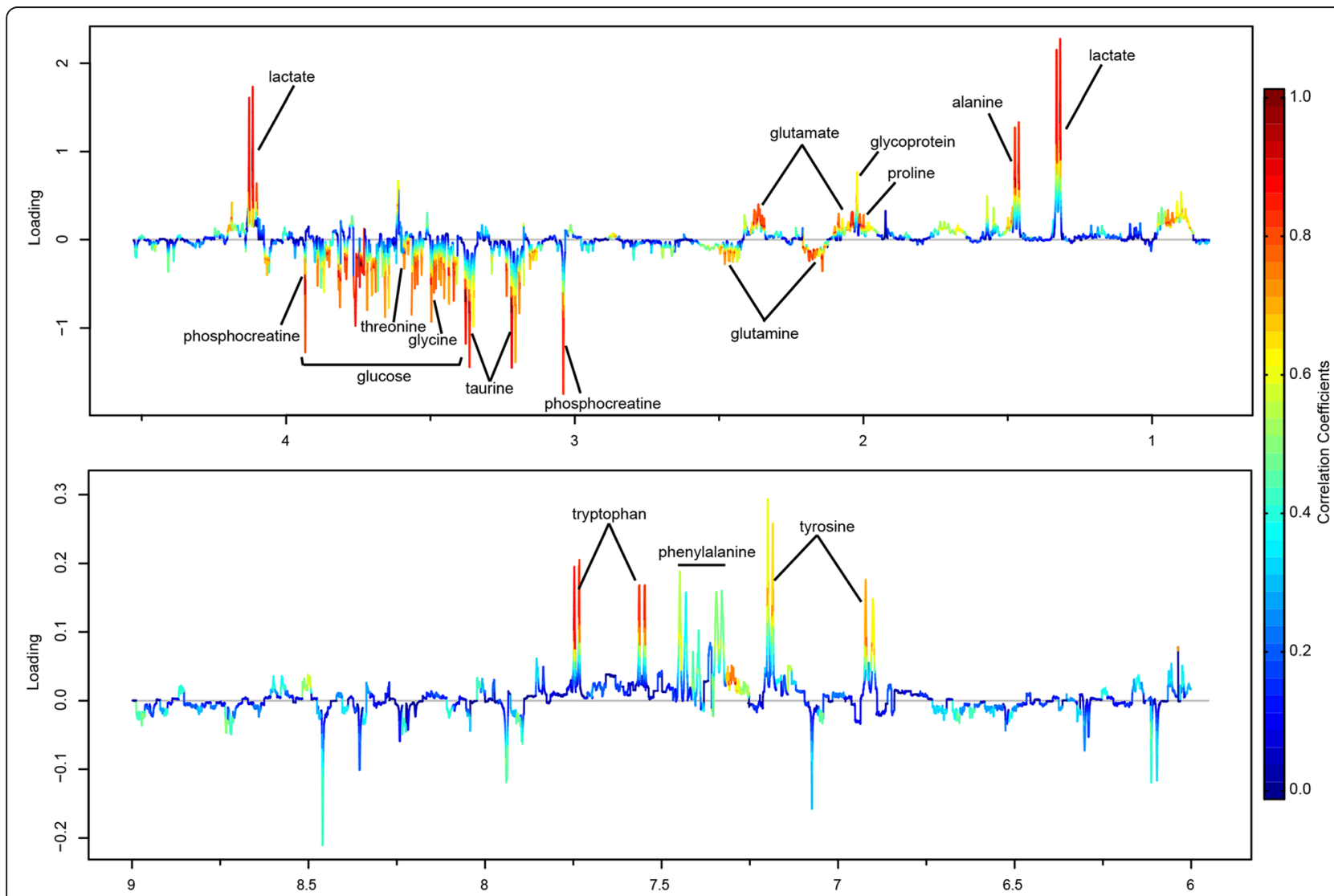

Fig. 3 Loadings plot of PLS-DA model color-coded with the absolute value of correlation coefficients. Positive peaks corresponding to metabolites that increased in NSCLC group, and negative regions corresponding to metabolites that decreased in NSCLC group 


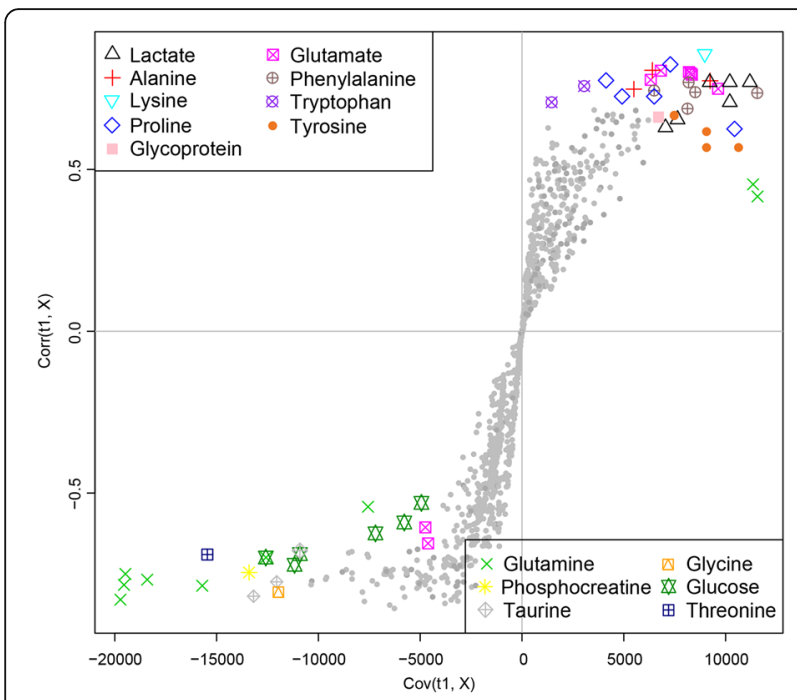

Fig. 4 S-plot from PLS-DA analysis to visualize the variable influences and to filter potential metabolites. Warm color (red) denotes a large contribution to grouping and cold color (blue) denotes less contribution to grouping. The significantly increased metabolites in NSCLC group were located in the upper-right quadrant and the decreased metabolites were located in the lower-left quadrant

taurine, glutamine, glycine, phosphocreatine and threonine were decreased, concerning several possible mechanisms of hyperthermic cell killing such as rupture of plasma membrane, dysfunction of mitochondrial, DNA damage, and energy-based cell death.

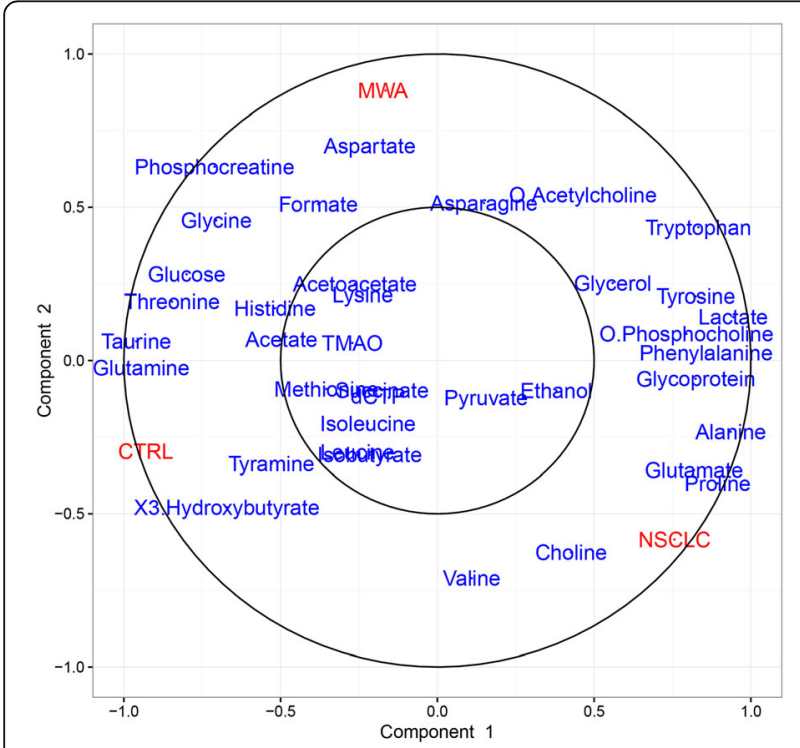

Fig. 5 Concentric correlation circle plot concerning the correlations between the identified metabolites and the three groups, provided additional information on the endogenous metabolites among groups

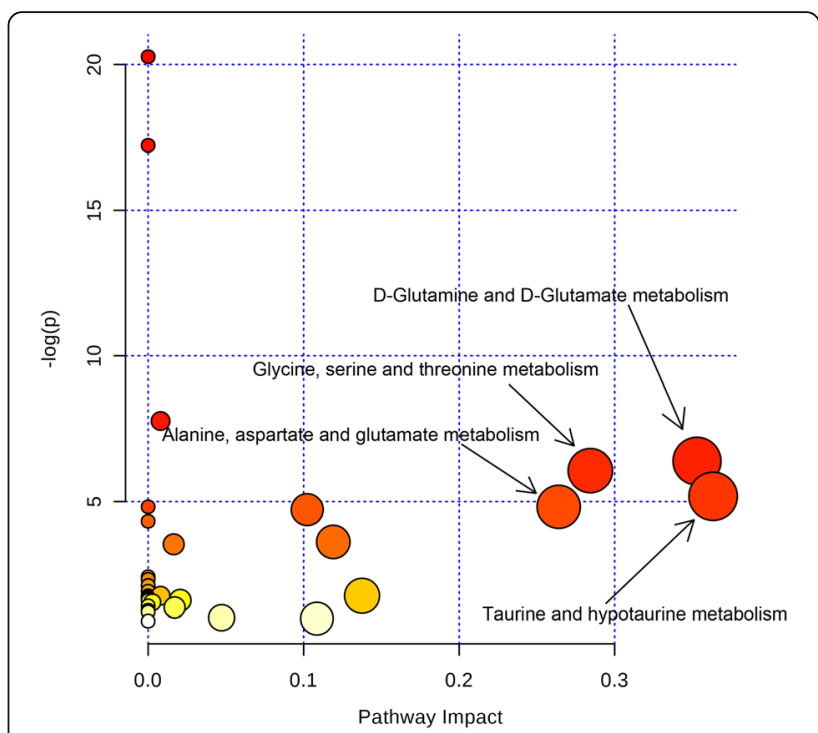

Fig. 6 Pathway topology analysis in association with NSCLC. Bubble area donating to the impact of each pathway, with color representing the significance from highest in red to lowest in white

\section{Destroy of membrane}

Hyperthermia induced by MWA have been shown to change cell membrane integrity and fluidity, which were first considered to be the main cause of cell death [5, 22, 23]. Phosphocholine and choline, components of the plasma membranes, are often regarded as markers of the integrity of membranes. In our experiment, the slightly lowered levels of choline and O-phosphocholine in NSCLC group indicated an accelerated use of them for cancer cell proliferation. Compared with NSCLC, their levels in MWA group have no significant change. Ablation change the fluidity and permeability of cell membrane, thereby leading to concretion and cytolysis, subsequently causing intracellular metabolites shifts.

\section{Dysfunction of mitochondrial}

Mitochondrial dysfunction has been well correlated with heat-induced injury [24]. Some ultrastructural changes was observed previously, such as dilatation of the mitochondria with rupture or loss of the cristae [24]. The level of taurine in NSCLC patients was decreased, which was in accordance with previous reports [11, 25-28]. As an antioxidant, taurine may be utilized as an antioxidant defense system against the oxidative stress involved in cancer process [29]. Ablation disrupted the mitochondrial membrane potential, resulting in the change in the redox status of cells [30], inducing cancer cell killing, thus leading to the reduced consumption of taurine.

\section{DNA injury}

Glycine was found to be decreased, which was in accordance with the previous study [31]. As a simple, 


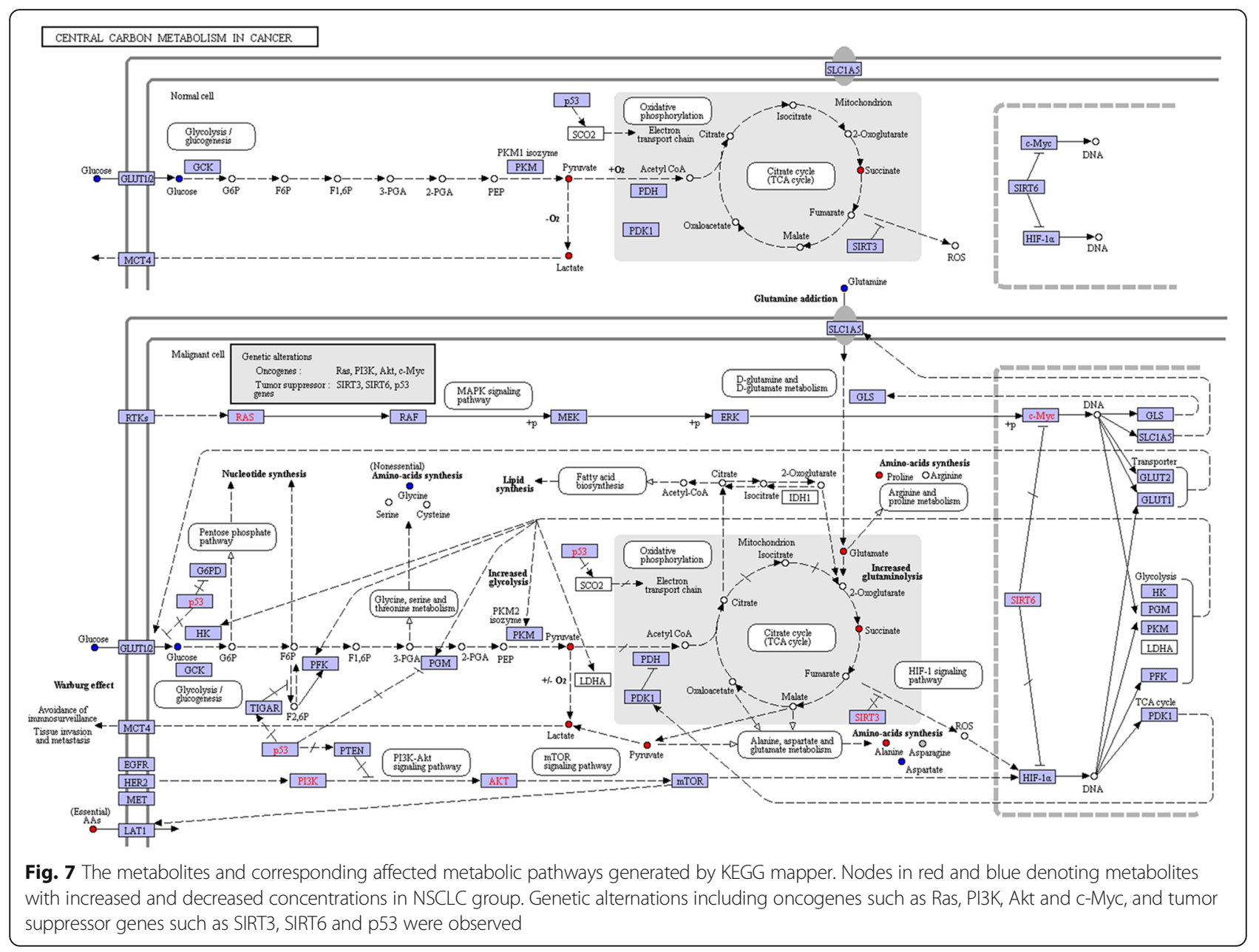

nonessential amino acid, glycine was involved in the production of DNA, phospholipids and collagen as well as the release of energy. Given that cancer cells reprogram their metabolisms comprehensively, the decreased level of glycine in NSCLC patients may be related with the accelerated DNA synthesis. The level of glycine was increased after hyperthermic ablation, indicating that hyperthermia treatment caused nucleotide damage. Previous research offers some support for this view, which indicated that hyperthermia could cause damage to DNA [30] and lead to inhibition of nucleolar RNA synthesis [24, 32, 33]. One possible mechanism of heat-induced DNA injury was the denaturation of some key replication enzymes, such as DNA polymerase $\alpha$ and $\beta$, which is responsible for DNA replication and repair synthesis, respectively [34]. Another potential mechanism could be attributed to the formation of endogenous reactive oxygen species (ROS) as a consequence of hyperthermic ablation [5], which subsequently results in DNA damage because DNA is particularly vulnerable to ROS-induced damage. As a result, imbalance of glycine related to DNA damage and repairs was found [35].

\section{Energy-based cell death}

The level of lactate was increased in NSCLC patients, which was in accordance with the previous studies [11, 31, 36-38]. As previously reported, cancer cell metabolism involves primarily the conversion of glucose ultimately to lactate by an enzyme-catalyzed anaerobic fermentation rather than the oxidation of glucose ultimately to carbon dioxide and water as occurs with normal cells, known as the Warburg effect [39]. Recent study has proposed that lactate is also a tricarboxylic acid (TCA) cycle carbon source for NSCLC and sustain tumor metabolism in vivo [40].Therefore, the elevated levels of lactate found in the serum of NSCLC patients could be attributed to the enormous domestic for cell proliferation. Hyperthermia caused extensive damage of the ultrastructural of mitochondria, ruptured glycolysis and energy metabolism of cancerous cells, which blocking the generation of lactate as a fuel of TCA cycle.

Levels of serum alanine was elevated in NSCLC group, which was consistent with previous report [36]. Alanine is a glycogenic (glycogen-producing) amino acid that can be converted to pyruvate and tricarboxylic acid cycle intermediates, and then to glucose by gluconeogenesis, 
functioning as an energy source to meet the huge demand of energy consumed in various metabolic activities in tumor cells. Elevated levels of serum alanine may facilitate energy synthesis in the cells and provide enough energy for cell growth. Tumor cells also utilized glutamine as another energy supply [41], which was called glutaminolysis. The decreased level of glutamine has been reported previously [42, 43], which was possibly due to its consumption for energy supply. After MWA treatment, the elevated level of alanine and decreased level of glutamine were recovered to the status of normal control group. Destroy of mitochondria and coagulative necrosis of cancer cells caused by hyperthermia might be responsible for the reversion of the above metabolites which are related with energy metabolism.

However, the roles of the significant pathways mentioned above in relation to MWA treatment were only hypothesis, without any complementary techniques as a backing. The model without vigorous explaination and prediction ability limits us to draw any definitive conclusions, which may be attributed to the small sample size and the heterogeneity of patients. Future studies with a larger sample size are planned to more clearly characterize metabolic profiles of NSCLC patients.

\section{Conclusions}

${ }^{1} \mathrm{H}$ NMR-based metabolomics approach could effectively distinguish the metabolic profile of NSCLC patients with or without MWA treatment from that of healthy controls. The observed potential biomarkers may facilitate to diagnose NSCLC non-invasively. MWA method could partially reverse the disturbed metabolic profile towards the control group. In the future, more patients from different ethnicities should be enrolled to verify the precision and specificity of the potential biomarkers in the diagnosis of NSCLC and the efficacy evaluation of MWA in curing NSCLC in clinic.

\section{Additional file}

Additional file 1: Table S1. Potential serum biomarkers identified by ${ }^{1} \mathrm{H}$ NMR and their means and standard deviation values for each group. Table S2. Pathway analysis and the altered pathways using MetaboAnalyst. Figure S1. PCA scores plot of ${ }^{1} \mathrm{H}$ NMR spectra from control, NSCLC and MWA groups. Figure S2. Scatter plots of the metabolites to show the interindividual variation. Gray circle: CTRL group, green circle: NSCLC group, red circle: MWA group. (DOCX $554 \mathrm{~kb})$

\footnotetext{
Abbreviations

AKT: Protein kinase B; c-Myc: MYC proto-oncogene; DNA: deoxyribonucleic acid; HDL: high density lipoprotein; HMDB: Human metabolites database; KEGG: Kyoto Encyclopedia of Genes and Genomes; LDL: low-density lipoprotein; MMCD: Madison Metabolomics Consortium Database; MWA: microwave ablation; NMR: nuclear magnetic resonance; NSCLC: nonsmall-cell lung cancer; PCA: Principal Component Analysis; PI3K: phosphatidylinositol 3kinase; PLS-DA: Partial least squares discriminant analysis; Ras: rat sarcoma; RNA: ribonucleic acid; ROS: Reactive Oxygen Species; SIRT3: sirtuin-3; SIRT6: Sirtuin 6; TCA: tricarboxylic acid; VLDL: very low density lipoprotein
}

\section{Acknowledgements}

Not applicable.

\section{Funding}

This research received no specific grant from any funding agency in the public, commercial, or not-for-profit sectors.

Availability of data and materials

Please contact author for data requests.

\section{Authors' contributions}

$\mathrm{JH}$ participated in the design of the study, performed the statistical analysis and write the manuscript. HS conceived of the study, and participated in its design and coordination and helped to draft the manuscript. Both authors read and approved the final manuscript.

Ethics approval and consent to participate

The study was approved by the Institutional Ethics Committee of the Jiangxi Provincial People's Hospital.

Consent for publication

Not applicable.

\section{Competing interests}

The authors declare that they have no competing interests.

\section{Publisher's Note}

Springer Nature remains neutral with regard to jurisdictional claims in published maps and institutional affiliations.

Received: 15 September 2017 Accepted: 22 February 2018 Published online: 12 March 2018

\section{References}

1. Chen W, Zheng R, Baade PD, Zhang S, Zeng H, Bray F, et al. Cancer statistics in China, 2015. CA Cancer J Clin. 2016;66(2):115-32.

2. Wei Z, Ye X, Yang X, Huang G, Li W, Wang J, et al. Advanced non small cell lung cancer: response to microwave ablation and EGFR status. Eur Radiol. 2016:1-10.

3. Chamarthy MR, Gupta M, Hughes TW, Velasco NB, Cynamon J, Golowa Y. Image-guided percutaneous ablation of lung malignancies: a minimally invasive alternative for nonsurgical patients or unresectable tumors. J Bronchol Interv Pulmonol. 2014;21:68-81.

4. Cha TS, Najihah MG, Bin Sahid I, Chuah TS. Molecular basis for resistance to ACCase-inhibiting fluazifop in Eleusine indica from Malaysia. Pestic Biochem Phys. 2014;111:7-13.

5. Chu KF, Dupuy DE. Thermal ablation of tumours: biological mechanisms and advances in therapy. Nat Rev Cancer. 2014;14:199-208.

6. Smith $S$, Jennings $P$. Lung radiofrequency and microwave ablation: a review of indications, techniques and post-procedural imaging appearances. $\mathrm{Br}$ J Radiol. 2015;88:20140598.

7. Zhang X, Zhu X, Wang C, Zhang H, Cai Z. Non-targeted and targeted metabolomics approaches to diagnosing lung cancer and predicting patient prognosis. Oncotarget. 2016;7:63437-48.

8. Puchades-Carrasco L, Jantus-Lewintre E, Perez-Rambla C, Garcia-Garcia F, Lucas R, Calabuig S, et al. Serum metabolomic profiling facilitates the noninvasive identification of metabolic biomarkers associated with the onset and progression of non-small cell lung cancer. Oncotarget. 2016;7:12904-16.

9. Chen W, Lu S, Ou J, Wang G, Zu Y, Chen F, et al. Metabonomic characteristics and biomarker research of human lung cancer tissues by HR1H NMR spectroscopy. Cancer Biomark. 2016;16:653-64.

10. Deja S, Porebska I, Kowal A, Zabek A, Barg W, Pawelczyk K, et al. Metabolomics provide new insights on lung cancer staging and discrimination from chronic obstructive pulmonary disease. J Pharm Biomed Anal. 2014;100:369-80

11. Rocha CM, Carrola J, Barros AS, Gil AM, Goodfellow BJ, Carreira IM, et al. Metabolic signatures of lung cancer in biofluids: NMR-based metabonomics of blood plasma. J Proteome Res. 2011;10:4314-24.

12. Hao D, Sarfaraz MO, Farshidfar F, Bebb DG, Lee CY, Card CM, et al. Temporal characterization of serum metabolite signatures in lung cancer patients undergoing treatment. Metabolomics. 2016;12:58. 
13. Xia J, Wishart DS. Web-based inference of biological patterns, functions and pathways from metabolomic data using MetaboAnalyst. Nat Protoc. 2011;6:743.

14. Bykov VJ, Issaeva N, Shilov A, Hultcrantz M, Pugacheva E, Chumakov P, et al. Restoration of the tumor suppressor function to mutant p53 by a lowmolecular-weight compound. Nat Med. 2002;8:282-8.

15. Haigis MC, Deng C-X, Finley LW, Kim H-S, Gius D. SIRT3 is a mitochondrial tumor suppressor: a scientific tale that connects aberrant cellular ROS, the Warburg effect, and carcinogenesis. Cancer Res. 2012;72:2468-72

16. Sebastián C, Zwaans BM, Silberman DM, Gymrek M, Goren A, Zhong L, et al. The histone deacetylase SIRT6 is a tumor suppressor that controls cancer metabolism. Cell. 2012;151:1185-99.

17. Coppé J-P, Patil CK, Rodier F, Sun Y, Muñoz DP, Goldstein J, et al. Senescenceassociated secretory phenotypes reveal cell-nonautonomous functions of oncogenic RAS and the p53 tumor suppressor. PLoS Biol. 2008;6:e301.

18. Leevers SJ, Weinkove D, MacDougall LK, Hafen E, Waterfield MD. The drosophila phosphoinositide 3-kinase Dp110 promotes cell growth. EMBO J. 1996;15:6584-94

19. Altomare DA, Testa JR. Perturbations of the AKT signaling pathway in human cancer. Oncogene. 2005;24:7455-64.

20. Hsu TY-T, Simon LM, Neill NJ, Marcotte R, Sayad A, Bland CS, et al. The spliceosome is a therapeutic vulnerability in MYC-driven cancer. Nature. 2015;525:384-8.

21. Dupuy DE. Treatment of medically inoperable non-small-cell lung cancer with stereotactic body radiation therapy versus image-guided tumor ablation: can interventional radiology compete? J Vasc Interv Radiol. 2013; 24:1139-45.

22. Bowler K. Cellular heat injury: are membranes involved? Symp Soc Exp Biol. 1987:41:157-85.

23. Hahn GM, Li GC, Shiu E. Interaction of amphotericin B and 43 degrees hyperthermia. Cancer Res. 1977;37:761-4.

24. Fajardo LF, Egbert B, Marmor J, Hahn GM. Effects of hyperthermia in a malignant tumor. Cancer. 1980;45:613-23.

25. Robaszkiewicz A, Bartosz G, Soszynski M. N-Chloroamino acids mediate the action of hypochlorite on A549 lung cancer cells in culture. Toxicology. 2010;270:112-20.

26. Zhang L, Jin H, Guo X, Yang Z, Zhao L, Tang S, et al. Distinguishing pancreatic cancer from chronic pancreatitis and healthy individuals by (1)H nuclear magnetic resonance-based metabonomic profiles. Clin Biochem. 2012;45:1064-9.

27. Gao H, Lu Q, Liu X, Cong $H$, Zhao L, Wang $H$, et al. Application of $1 H$ NMRbased metabonomics in the study of metabolic profiling of human hepatocellular carcinoma and liver cirrhosis. Cancer Sci. 2009;100:782-5.

28. Hasim A, Ali M, Mamtimin B, Ma JQ, Li QZ, Abudula A. Metabonomic signature analysis of cervical carcinoma and precancerous lesions in women by (1)H NMR spectroscopy. Exp Ther Med. 2012;3:945-51.

29. Reuter S, Gupta SC, Chaturvedi MM, Aggarwal BB. Oxidative stress, inflammation, and cancer: how are they linked? Free Radic Biol Med. 2010;49:1603-16.

30. Roti Roti JL. Cellular responses to hyperthermia (40-46 degrees C): cell killing and molecular events. Int J Hyperth. 2008;24:3-15.

31. Rocha CM, Barros AS, Gil AM, Goodfellow BJ, Humpfer E, Spraul M, et al. Metabolic profiling of human lung cancer tissue by $1 \mathrm{H}$ high resolution magic angle spinning (HRMAS) NMR spectroscopy. J Proteome Res. 2010;9:319-32

32. Simard R, Bernhard W. A heat-sensitive cellular function located in the nucleolus. J Cell Biol. 1967;34:61-76

33. Mondovi B, Strom R, Rotilio G, Finazzi Agro A, Cavaliere R, Rossi Fanelli A. The biochemical mechanism of selective heat sensitivity of cancer cells. I. Studies on cellular respiration. Eur J Cancer. 1969:5:129-36.

34. Warters RL, Roti JLR. Hyperthermia and the cell-nucleus. Radiat Res 1982;92: 458-462.

35. Menon SS, Uppal M, Randhawa S, Cheema MS, Aghdam N, Usala RL, et al. Radiation metabolomics: current status and future directions. Front Oncol. 2016;6:20.

36. Fan TW, Lane AN, Higashi RM, Farag MA, Gao H, Bousamra M, et al. Altered regulation of metabolic pathways in human lung cancer discerned by (13)C stable isotope-resolved metabolomics (SIRM). Mol Cancer. 2009;8:41.

37. Jordan KW, Adkins CB, Su L, Halpern EF, Mark EJ, Christiani DC, et al. Comparison of squamous cell carcinoma and adenocarcinoma of the lung by metabolomic analysis of tissue-serum pairs. Lung Cancer. 2010;68:44-50.
38. Altenberg B, Greulich KO. Genes of glycolysis are ubiquitously overexpressed in 24 cancer classes. Genomics. 2004:84:1014-20.

39. Garber K. Energy boost: the Warburg effect returns in a new theory of cancer. J Natl Cancer Inst. 2004;96:1805-6.

40. Faubert B, Li KY, Cai L, Hensley CT, Kim J, Zacharias LG, et al. Lactate metabolism in human lung tumors. Cell. 2017;171:358-71. e9.

41. Deberardinis RJ, Sayed N, Ditsworth D, Thompson CB. Brick by brick: metabolism and tumor cell growth. Curr Opin Genet Dev. 2008;18:54-61.

42. Bathe OF, Shaykhutdinov R, Kopciuk K, Weljie AM, McKay A, Sutherland FR, et al. Feasibility of identifying pancreatic cancer based on serum metabolomics. Cancer Epidemiol Biomark Prev. 2011;20:140-7.

43. Kubota A, Meguid MM, Hitch DC. Amino acid profiles correlate diagnostically with organ site in three kinds of malignant tumors. Cancer. 1992;69:2343-8.

\section{Submit your next manuscript to BioMed Central and we will help you at every step:}

- We accept pre-submission inquiries

- Our selector tool helps you to find the most relevant journal

- We provide round the clock customer support

- Convenient online submission

- Thorough peer review

- Inclusion in PubMed and all major indexing services

- Maximum visibility for your research

Submit your manuscript at www.biomedcentral.com/submit 